\title{
Efficacy and safety profile of doxofylline compared to theophylline in asthma: a meta-analysis
}

\author{
Paola Rogliani', Luigino Calzetta ${ }^{1 *} \mathbb{D}$, Josuel Ora², Mario Cazzola and Maria Gabriella Matera ${ }^{3}$
}

\begin{abstract}
Background: Oral methylxanthines are effective drugs for the treatment of chronic obstructive respiratory disorders. The novel methylxanthine doxofylline, that has bronchodilator and anti-inflammatory activities, is not affected by the major drawback of theophylline. Nowadays large-scale quantitative synthesis comparing the efficacy and safety profile of doxofylline vs. theophylline in the treatment of asthma is still lacking. Therefore, we performed a quantitative synthesis to compare the efficacy/safety profile of doxofylline and theophylline in asthma.
\end{abstract}

Methods: A pairwise and network meta-analyses were performed to assess the impact of doxofylline vs. theophylline and placebo on the change in asthma events, risk of adverse events (AEs), forced expiratory volume in $1 \mathrm{~s}\left(\mathrm{FEV}_{1}\right)$, and salbutamol use.

Results: Data obtained from 696 asthmatic patients were extracted from 4 randomized controlled trials published between 2015 and 2018. Doxofylline was significantly $(P<0.05)$ more effective than theophylline in reducing the daily asthma events (mean difference $-0.14,95 \% \mathrm{Cl}-0.27-0.00$ ) and risk of AEs (relative risk $0.76,95 \% \mathrm{Cl} 0.59-0.99$ ). Doxofylline was as effective as theophylline in improving $\mathrm{FEV}_{1}$, and a trend of superiority $(P=0.058)$ was detected for doxofylline over theophylline with respect to the reduction in the use of salbutamol as rescue medication. The rank of effectiveness was doxofylline>theophylline > > placebo, and the rank of safety was placebo>doxofylline>> theophylline.

Conclusions: Doxofylline is an effective and safe methylxanthine for the treatment of asthma, with an efficacy/ safety profile greater than that of theophylline.

Trial registration: Meta-analysis registration: CRD42019119849.

Keywords: Asthma, Doxofylline, Theophylline, Meta-analysis

\section{Background}

Oral methylxanthines are recognized effective drugs for the clinical management of patients suffering from chronic obstructive respiratory disorders. The novel methylxanthine doxofylline, that is characterized by bronchodilator and antiinflammatory activities, seems to be not affected by the major drawback of theophylline [1-5]. Doxofylline has a favourable efficacy profile accompanied by a high level of tolerability, at least in COPD patients [6]. Moreover, an observational study aimed to assess the treatment plan in acute

\footnotetext{
* Correspondence: luigino.calzetta@uniroma2.it

'Unit of Respiratory Medicine, Department of Experimental Medicine, University of Rome "Tor Vergata", Via Montpellier 1, 00133 Rome, Italy Full list of author information is available at the end of the article
}

and chronic respiratory tract diseases, has demonstrated a significant difference in the unit cost per patient in favour of doxophylline compared to theophyllne. In fact, although the cost of doxophylline was higher than that of theophyllne, doxofylline resulted to be associated with a reduction of the overall cost related with COPD and/or asthma management [7].

Recently, the pooled analysis of two multicenter, double-blind, randomized trials, carried out in 38 US pulmonary clinics that investigated the therapeutic efficacy and tolerability of doxofylline compared to theophylline, demonstrated that doxofylline is an effective and well tolerated agent in asthmatic patients [8]. Although the beneficial efficacy/safety profile of doxofylline

(c) The Author(s). 2019 Open Access This article is distributed under the terms of the Creative Commons Attribution 4.0 International License (http://creativecommons.org/licenses/by/4.0/), which permits unrestricted use, distribution, and 
has been reported in further smaller clinical trials and retrospective studies [9-14], to date large-scale quantitative synthesis comparing the efficacy and safety profile of doxofylline vs. theophylline in the treatment of asthma is still lacking.

The hypothesis of this study is that a quantitative synthesis of clinical trials that directly compared the efficacy and safety of doxofylline vs. theophylline may provide more robust evidences than individual studies or pooled analyses. Therefore, we performed a pairwise and network meta-analysis to definitively clarify which of the two drugs should be prescribed when a methylxanthine is recommended in asthmatic patients.

\section{Methods}

\section{Search strategy}

This meta-analysis has been submitted to the international database of prospectively registered systematic reviews (PROSPERO, registration number: CRD42019119849), and performed in agreement with the Preferred Reporting Items for Systematic Reviews and Meta-Analyses Protocols (PRISMA-P) [15]. The PRISMA flow diagram is reported in Fig. 1a. This quantitative synthesis satisfied all the recommended items reported by the PRISMA-P checklist [15].

Two reviewers performed a comprehensive literature search for randomized controlled trials (RCTs) evaluating the influence of doxofylline and theophylline in adolescent and adult asthmatic patients. The PICO (Patient problem, Intervention, Comparison, and Outcome) framework was used to develop the literature search strategy, as previously described [16]. Namely, the "Patient problem" included subject affected by asthma; the "Intervention" regarded the administration of doxofylline and theophylline; the "Comparison" was performed with regard to placebo and across each active treatment; the "Outcomes" were the asthma events, adverse events (AEs), lung function expressed as forced expiratory volume in $1 \mathrm{~s}\left(\mathrm{FEV}_{1}\right)$, and use of salbutamol as rescue medication.

The terms "doxofylline" AND "theophylline" AND "asthma" were searched in Cochrane Central Register of Controlled Trials (CENTRAL), MEDLINE, Embase, Scopus, Web of Science, ClinicalTrials.gov and EU Clinical Trials Register databases in order to provide for relevant studies available up to December 7, 2018. No language restriction was applied. The following Query Translation was used: "asthma" [MeSH Terms] OR "asthma"[All Fields]) AND ("theophylline" [MeSH Terms] OR "theophylline" [All Fields]) AND ("doxofylline" [Supplementary Concept] OR "doxofylline"[All Fields]).

Citations of previously published analyses and relevant reviews were examined to identify further pertinent studies, if any $[6,8,17-19]$.

\section{Study selection}

Published RCTs involving asthmatic patients treated with oral formulations of doxofylline and theophylline were included in this meta-analysis.

Two reviewers independently checked the relevant studies identified from literature searches obtained from

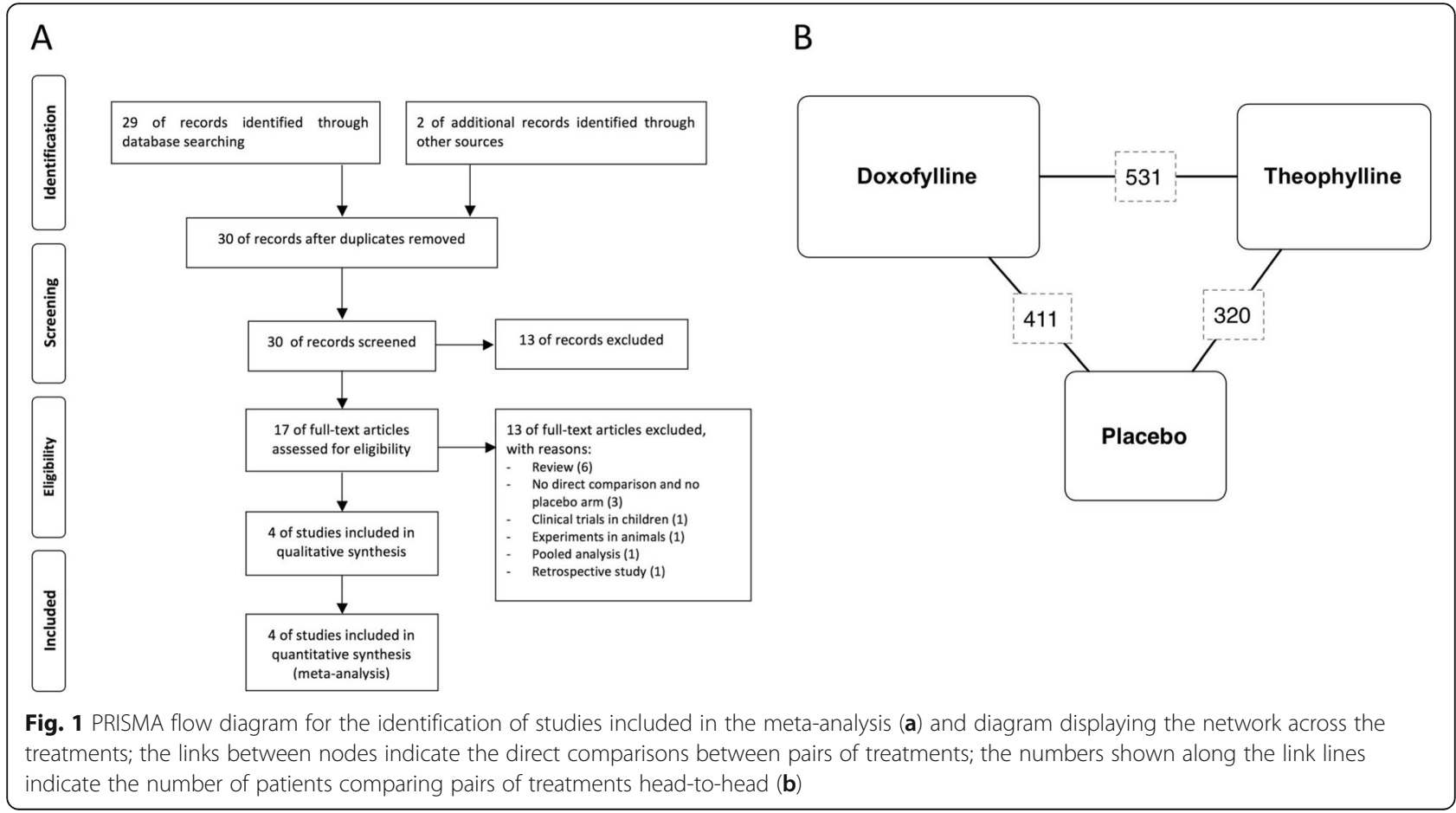


the already mentioned databases. The studies were selected in agreement with the above-mentioned criteria, and any difference in opinion about eligibility was resolved by general consensus.

\section{Endpoints}

The primary endpoints of this meta-analysis were the impact of doxofylline on the change in asthma events and the risk of AEs, compared to theophylline and placebo. Asthma events were defined as episodes of increased symptoms or increased airflow limitation responsive to as needed shortacting $\beta_{2}$ agonists or resulting in either unscheduled medical attention, unscheduled use of oral corticosteroids, hospital admission [20]. The secondary endpoints were the impact of doxofylline on the change in $\mathrm{FEV}_{1}$ and salbutamol use, compared to theophylline.

\section{Quality score, risk of bias and evidence profile}

The Jadad score, with a scale of 1 to 5 (score of 5 being the best quality), was used to assess the quality of the RCTs concerning the likelihood of biases related to randomization, double blinding, withdrawals and dropouts [21]. A Jadad score $\geq 3$ was defined to identify high quality studies. Two reviewers independently assessed the quality of individual studies, and any difference in opinion about the quality score was resolved by consensus.

In the pairwise meta-analysis moderate to high levels of heterogeneity between-studies were considered for $\mathrm{I}^{2}>$ $50 \%$; the risk of publication bias was assessed by applying the funnel plot and Egger's test, as previously described [21]. Evidence of asymmetry from Egger's test was considered to be significant at $P<0.1$, and the graphical representation of $90 \%$ confidence bands have been presented [21]. The risk of bias in the network meta-analysis was checked via the consistency/inconsistency analysis to assess whether the outcomes resulting from the consistency and inconsistency models fit adequately with the line of equality, as previously described [22].

The quality of the evidence was assessed for the primary endpoint in agreement with the Grading of Recommendations Assessment, Development, and Evaluation (GRADE) system, indicating ++++ for high quality of evidence, +++ for moderate quality of evidence, ++ for low quality of evidence, and + for very low quality of evidence [23].

\section{Data extraction}

Data from included RCTs were extracted and checked for study characteristics and duration, enrolled patients, treatments and doses, disease characteristics, age, gender, asthma events, previous hospitalizations, lung function, and Jadad score. Data have been extracted in agreement with DECiMAL recommendations [24].

\section{Data analysis}

A pairwise meta-analysis was performed to quantify the impact of doxofylline compared to theophylline on primary and secondary endpoints.

Results of the pairwise meta-analysis are expressed as mean difference (MD) or relative risk (RR), and 95\% confidence interval $(95 \% \mathrm{CI})$. Since data were selected from a series of studies performed by researchers operating independently, and a common effect size cannot be assumed, binary random-effects model was used in order to balance the study weights and adequately estimate the $95 \% \mathrm{CI}$ of the mean distribution of drugs effect on the investigated variables [21].

A network meta-analysis was also carried out to perform a comparison across doxofylline, theophylline, and placebo with respect to the primary endpoints, and to rank their efficacy in reducing asthma events and the risk of AEs.

The network meta-analysis was carried out by including RCTs that introduced no significant heterogeneity and bias in the effect estimates of primary endpoint. Since heterogeneity and bias may propagate through a network of RCTs, and thus affect the estimates differentially across regions of the network, this approach permitted to identify those studies that might alter the correct results of the network meta-analysis [25].

Full Bayesian evidence network was used in the network meta-analysis (chains: 4 ; initial values scaling: 2.5 ; tuning iterations: 20.000; simulation iterations: 50.000; tuning interval: 10). The convergence diagnostics for consistency and inconsistency was assessed via the Brooks-GelmanRubin method, as previously described [26]. Results of the network meta-analysis are expressed as relative effect (RE) and $95 \%$ credible interval $(95 \% \mathrm{CrI})$. The probability that each intervention arm was the most effective was calculated by counting the proportion of iterations of the chain in which each intervention arm had the highest mean difference, and the surface under the cumulative ranking curve (SUCRA), representing the summary of these probabilities, was also calculated. The SUCRA is 1 when a treatment is certain to be the best, and 0 when a treatment is certain to be the worst [27].

Sensitivity analysis was performed to identify the studies that introduced heterogeneity and bias in the effect estimate of primary endpoints, if any. Meta-regression analysis was performed to identify the factors that might modulate the efficacy and safety profile of the investigated agents with regard to primary endpoints.

The specisafety profile of the investigated treatments was investigated through a pooled analysis of the frequency of specific AEs.

OpenMetaAnalyst [28] and GeMTC [29] software were used for performing the meta-analysis, GraphPad Prism (CA, US) software to graph the data, and GRADEpro GDT to assess the quality of evidence [23]. The statistical significance for 
the effect estimates resulting from the pairwise and network meta-analyses was assessed for $P<0.05$.

\section{Results}

\section{Studies characteristics}

Data obtained from 696 asthmatic patients (44.68\% treated with doxofylline, $31.62 \%$ treated with theophylline, and $23.71 \%$ treated with placebo) were selected from 4 RCTs published between 2015 and 2018 [8-10]. The relevant characteristics of studies, disease, and patients are described in Table 1; Fig. 1b shows the network across the treatments.

All the RCTs were published as full-text papers [8-10]. The DOROTHEO 1, DOROTHEO 2, and the study of Margay et al. were published as high quality studies (Jadad score $\geq 3)[8,9]$, whereas the study of Lal et al. as lowquality study (Jadad score $=1$ ) [10]. The duration of treatment ranged from 6 weeks to 12 weeks.

\section{Primary endpoints}

The pairwise meta-analysis indicated that doxofylline was significantly $(P<0.05)$ more effective than theophylline in reducing the daily asthma events $(\mathrm{MD}-0.14,95 \% \mathrm{CI}-0.27$ - 0.00) (Fig. 2a). The effect estimate was not affected by heterogeneity $\left(\mathrm{I}^{2} 0 \%, P=0.53\right)$. Although a certain level of asymmetry resulted by the visual inspection of funnel plot, the Egger's test found not significant publication bias (Fig. $2 \mathrm{~b}$ and $\mathrm{c})$. The risk of AEs was significantly $(P<0.05)$ lower in patients treated with doxofylline than in those that received theophylline (RR $0.76,95 \% \mathrm{CI} \quad 0.59-0.99$ ) (Fig. 2d). The substantial but not significant heterogeneity $\left(\mathrm{I}^{2} 59 \%, P=0.05\right)$ was confirmed by the visual inspection of funnel plot (Fig. 2e), and mainly related with the study of Lal et al. [10]. However, the analysis performed via Egger's test indicated that, although the study of Lal et al. [10] was small and characterized by low quality, it introduced no significant publication bias in the effect estimate concerning the risk of AEs (Fig. 2f).

The results of the network meta-analysis indicated that doxofylline, but not theophylline, significantly $(P<0.05)$ reduced the daily asthma events (RE: $-0.33,95 \% \mathrm{CrI}-0.62$ $-0.04,-0.33$; -0.23 95\%CrI $-0.54-0.04$; respectively, compared to placebo). Doxofylline was also safer than theophylline, reporting a significant $(\mathrm{P}<0.05)$ reduction in the risk of AEs (RE 0.53, 95\%CrI 0.27-0.87; doxofylline vs. theophylline). The network meta-analysis also showed that doxofyline was the most effective treatment (upper quartile in the SUCRA ranking) with respect to both theophylline (second quartile in the SUCRA ranking) and placebo (lower quartile in the SUCRA ranking) (Fig. 2 g). As expected, placebo was the safest arm (upper quartile in the SUCRA ranking), followed by doxofylline (second quartile in the SUCRA ranking); conversely, theophylline was the less safe treatment (lower quartile in the SUCRA ranking) (Fig. 2 h).
The consistency/inconsistency analysis showed that all points fit adequately with the line of equality (efficacy: $\mathrm{R}^{2}$ 0.99 ; slope $1.05,95 \% \mathrm{CI} 0.96-1.15)$, indicating that the network meta-analysis was not affected by significant bias.

Meta-regression analysis confirmed that the patient demographics, baseline and study characteristics did not modulate the efficacy and safety profile of the investigated agents.

The GRADE analysis indicated high quality of evidence $(++++)$ for doxofylline vs. theophylline with respect to efficacy and safety profile in both pairwise and network meta-analysis.

\section{Secondary endpoints}

There was no significant difference between doxofylline and theophylline on the change in change in $\mathrm{FEV}_{1}(P>0.05)$ (Fig. 3a). A trend of superiority $(P=0.058)$ was detected for doxofylline over theophylline with respect to the reduction in the use of salbutamol as rescue medication (Fig. 3b).

The pooled analysis of safety profile showed that the AEs with a frequency greater than $5 \%$ were headache (doxofylline $20.61 \%$, theophylline $23.64 \%$ ), nausea (doxofylline $10.96 \%$, theophylline $21.82 \%$ ), nervousness (doxofylline $4.39 \%$, theophylline $11.36 \%$ ), and dyspepsia (doxofylline 6.58\%, theophylline 8.18\%). AEs were generally mild in severity, and detailed frequencies of further specific AEs are reported in Table 2.

\section{Discussion}

This meta-analysis showed that treatment with doxofylline was significantly more effective than theophylline in reducing the daily asthma events and preventing the risk of AEs, which were the primary endpoints of this metaanalysis. As expected, the SUCRA analysis performed by considering high quality RCTs reported that doxofylline had a better efficacy profile than both theophylline and placebo, and that theophylline was ranked as the less safe treatment in this quantitative synthesis. Interestingly, the pooled analysis indicated that the percentage of the most frequently recorded AEs (i.e. headache, nausea, nervousness, insomnia, dyspepsia, and vomiting) was generally greater in asthmatic patients treated with theophylline than in those that received doxofylline. Overall, the results of this study are strong, as they were not affected by publication bias, and with high quality of evidence for both the pariwise and network meta-analyses.

Concerning the secondary endpoints, doxofylline was as effective as theophylline in improving $\mathrm{FEV}_{1}$, although a trend toward significance [30] suggested that doxofylline was superior than theophylline concerning the reduction in the use of salbutamol as rescue medication.

Although significant, the difference in efficacy outcomes between doxofylline and theophylline did not reach the minimum clinically important differences (MCIDs), when considering the comparison across active treatments [31]. 


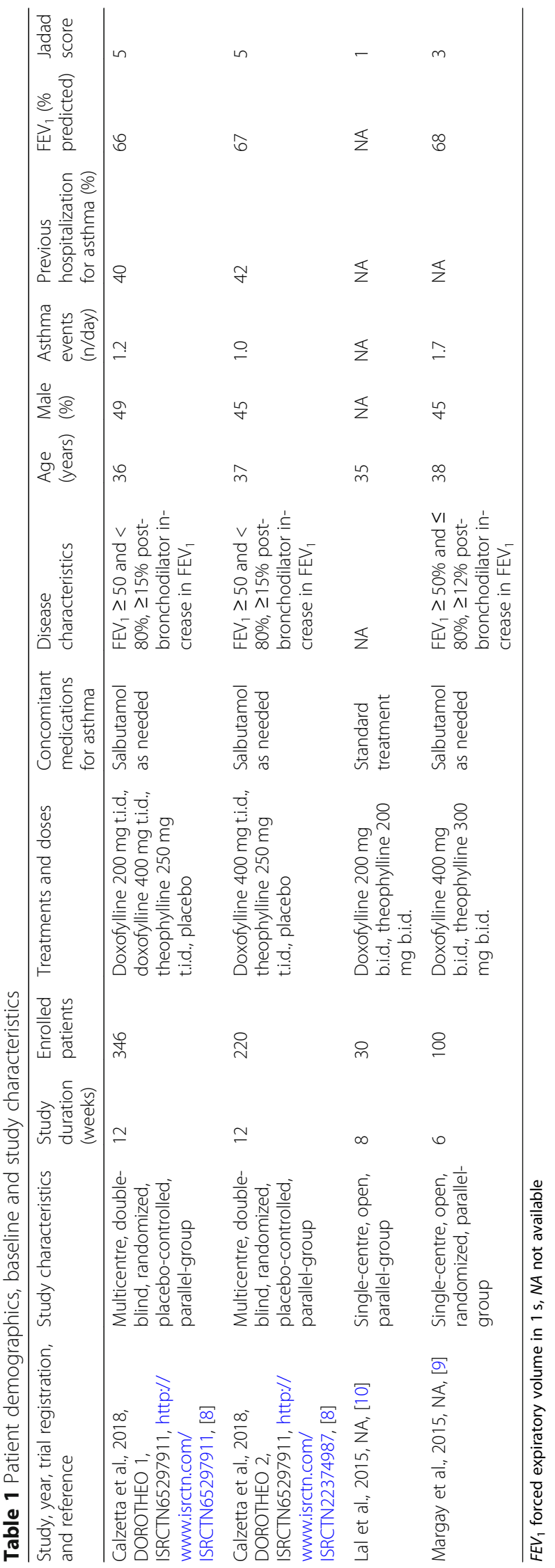



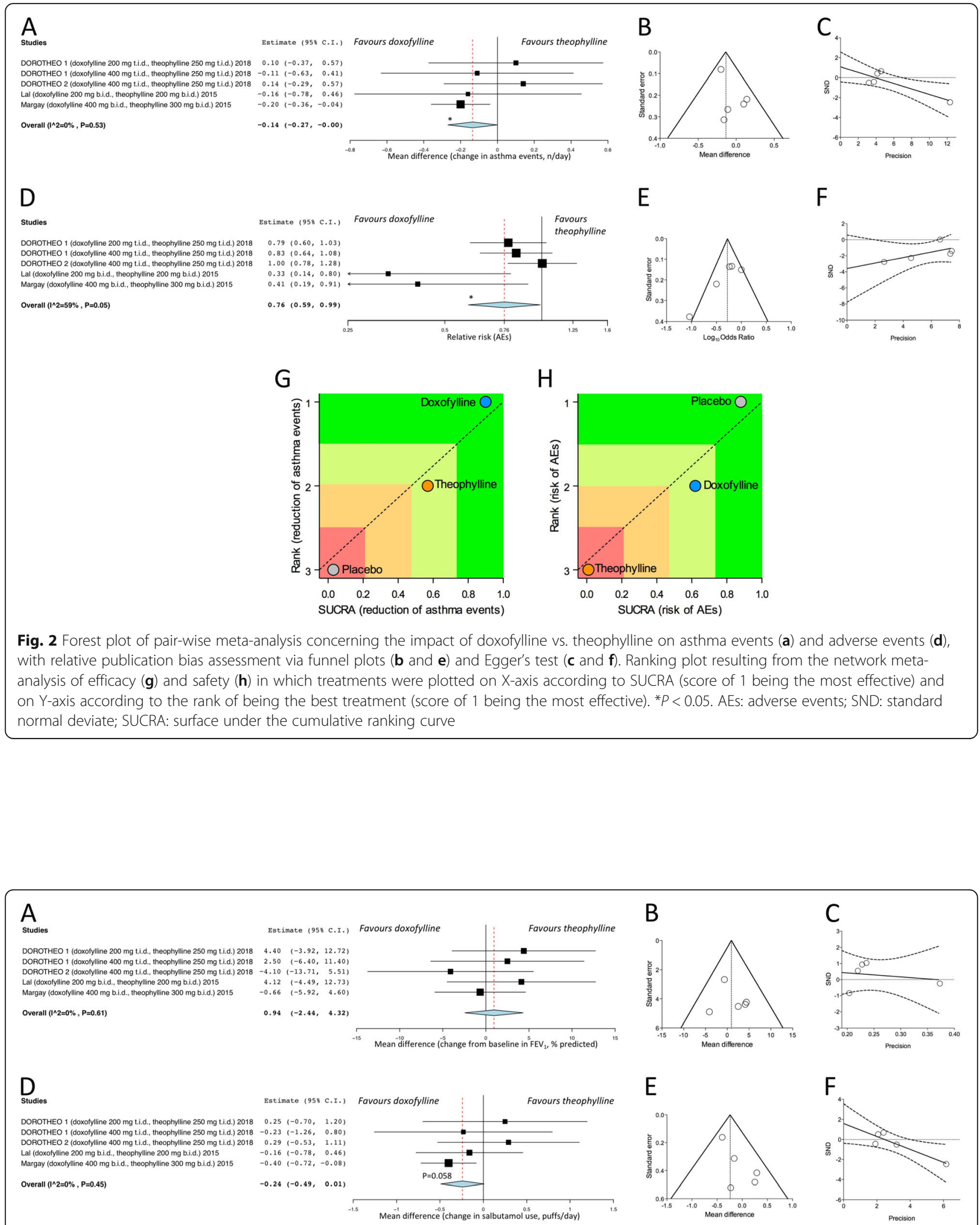

Fig. 3 Forest plot of pair-wise meta-analysis concerning the impact of doxofylline vs. theophylline on the change in FEV $V_{1}(\mathbf{a})$ and salbutamol use as recue medication (d), with relative publication bias assessment via funnel plots ( $\mathbf{b}$ and $\mathbf{e}$ ) and Egger's test (c and $\mathbf{f}$ ). FEV $\mathrm{V}_{1}$ : forced expiratory volume in $1 \mathrm{~s}$; SND: standard normal deviate 
Table 2 Pooled analysis of AEs (sorted by descending order) extracted from the studies that directly compared doxofyline with theophylline in asthmatic patients

\begin{tabular}{|c|c|c|c|c|}
\hline \multirow{3}{*}{ Total number of asthmatic patients } & \multirow{2}{*}{\multicolumn{2}{|c|}{$\begin{array}{l}\text { Doxofylline } \\
228 \\
\end{array}$}} & \multirow{2}{*}{\multicolumn{2}{|c|}{$\begin{array}{l}\text { Theophylline } \\
220\end{array}$}} \\
\hline & & & & \\
\hline & $\%$ & $\mathrm{n}$ & $\%$ & $\mathrm{n}$ \\
\hline Asthmatic patients reporting at least one $\mathrm{AE}$ & 46.05 & 105 & 57.73 & 127 \\
\hline \multicolumn{5}{|l|}{ Total number of AEs } \\
\hline headache & 20.61 & 47 & 23.64 & 52 \\
\hline nausea & 10.96 & 25 & 21.82 & 48 \\
\hline nervousness & 4.39 & 10 & 11.36 & 25 \\
\hline insomnia & 6.14 & 14 & 9.55 & 21 \\
\hline dyspepsia & 6.58 & 15 & 8.18 & 18 \\
\hline vomiting & 3.51 & 8 & 4.09 & 9 \\
\hline dizziness & 3.95 & 9 & 3.18 & 7 \\
\hline cough increased & 0.44 & 1 & 3.64 & 8 \\
\hline overdose & 0.00 & 0 & 3.64 & 8 \\
\hline rhinitis & 3.51 & 8 & 2.73 & 6 \\
\hline diarrhoea & 3.07 & 7 & 3.18 & 7 \\
\hline asthma & 3.07 & 7 & 3.18 & 7 \\
\hline abdominal pain & 2.63 & 6 & 1.82 & 4 \\
\hline pharyngitis & 1.32 & 3 & 2.27 & 5 \\
\hline palpitations & 2.19 & 5 & 1.82 & 4 \\
\hline chest pain & 2.19 & 5 & 0.00 & 0 \\
\hline epigastric distress & 0.88 & 2 & 1.82 & 4 \\
\hline asthenia & 0.00 & 0 & 1.82 & 4 \\
\hline tremors & 0.00 & 0 & 0.91 & 2 \\
\hline somnolence & 0.44 & 1 & 0.45 & 1 \\
\hline sore throat & 0.00 & 0 & 0.45 & 1 \\
\hline
\end{tabular}

$A E$ adverse event

Nevertheless, in the network meta-analysis the range of protection against the risk of AEs was clinically meaningful for doxofylline vs. theophylline, as the $95 \% \mathrm{CrI}$ of $\mathrm{RR}$ was prevalently below the 0.75 value, with the mean set at RR 0.53 [32]. The small differences in results between the pairwise and network meta-analyses may be due to the presence of the placebo node in the Bayesian analysis that reinforced the comparison across the investigated arms.

Taken together the findings of this meta-analysis support the rationale for using doxofyilline to treat chronic obstructive respiratory disorders, and its superiority with respect to theophylline $[6,8,17]$. Considering also the beneficial cost-effectiveness profile of the pharmacological treatment with doxofyilline [7], and the advantage of not needing the monitoring of theophyllinemia, there is no reason not to choose doxofylline as first line treatment when a methylxanthine is indicated in asthmatic patients.
Indeed, meta-analyses have evolved as a technique useful for summarizing the evidences form a large number of RCTs and for resolving discrepancies raised by clinical studies. Nevertheless, meta-analyses mainly deal with populations and not with single patients [33]. In this respect, the use of an effective, safe and inexpensive orally active drug as doxofylline should be encouraged especially in those patients who find inhalers difficult to use. The use of doxofylline, instead of theophylline, may have a strong rationale also in those patients who do not get adequate control from other pharmacological classes, such as inhaled corticosteroids in smokers asthmatics or $\beta_{2}$-adrenoceptor agonists in subjects with a genetic polymorphism resulting in homozygosity for arginine at amino acid residue 16 of the $\beta_{2}$-adrenergic receptor [34, 35].

Finally, but not less important, the current evidence clearly indicates that the Global Initiative for Asthma (GINA) recommendations [36] should be updated by considering doxofylline as a more effective and safer alternative to theophylline in Step 1 to 4 treatments, and as optional treatment in acute care settings.

\section{Conclusions}

The results of this quantitative synthesis of the current literature proves that doxofylline is an effective and safe methylxanthine for the treatment of asthma, and that its efficacy/safety profile is greater than that of theophylline.

\section{Abbreviations \\ AEs: adverse events; CENTRAL: Cochrane Central Register of Controlled Trials; $\mathrm{Cl}$ : confidence interval; $\mathrm{Crl}$ : credible interval; $\mathrm{FEV}_{1}$ : forced expiratory volume in $1 \mathrm{~s}$; GINA: Global Initiative for Asthma; GRADE: Grading of Recommendations Assessment, Development, and Evaluation; MD: mean difference; PICO: Patient problem, Intervention, Comparison, and Outcome; PRISMA- P: Preferred Reporting Items for Systematic Reviews and Meta-Analyses Pro- tocols; PROSPERO: prospectively registered systematic reviews; RCTs: randomized controlled trials; RE: relative effect; RR: relative risk; SUCRA: surface under the cumulative ranking curve}

Acknowledgements

Not applicable.

\section{Authors' contributions}

PR and LC: designed and executed the study, analyzed and interpreted the data, prepared the manuscript and approved the final version of the manuscript; JO: interpreted the data, prepared the manuscript and approved the final version of the manuscript; MC and MGM: designed the study, interpreted the data, prepared the manuscript and approved the final version of the manuscript. All authors read and approved the final manuscript.

\section{Funding}

ABC Farmaceutici (Italy) has provided unconditional support and funding for this research.

\section{Availability of data and materials}

All data generated or analysed during this study are included in this published article.

Ethics approval and consent to participate Not applicable. 


\section{Consent for publication}

Not applicable.

\section{Competing interests}

PR has participated as an advisor in scientific meetings the sponsorship of ABC Farmaceutici; LC has participated as an advisor in scientific meetings the sponsorship of $A B C$ Farmaceutici; he is or has been a consultant to $A B C$ Farmaceutici; JO has no competing interests; MGM has participated as an advisor in scientific meetings the sponsorship of ABC Farmaceutici; MC has participated as an advisor in scientific meetings the sponsorship of $A B C$ Farmaceutici; he is or has been a consultant to ABC Farmaceutici. MC is a member of the Editorial Board of Multidisciplinary Respiratory Medicine.

\section{Author details}

${ }^{1}$ Unit of Respiratory Medicine, Department of Experimental Medicine, University of Rome "Tor Vergata", Via Montpellier 1, 00133 Rome, Italy. 2Division of Respiratory Medicine, University Hospital "Tor Vergata", Rome, Italy. "Unit of Pharmacology, Department of Experimental Medicine, University of Campania "Luigi Vanvitelli", Naples, Italy.

\section{Received: 22 February 2019 Accepted: 29 May 2019}

Published online: 03 August 2019

\section{References}

1. Page CP. Doxofylline: a "novofylline". Pulm Pharmacol Ther. 2010;23(4):231-4.

2. Franzone JS, Cirillo R, Barone D. Doxofylline and theophylline are xanthines with partly different mechanisms of action in animals. Drugs Exp Clin Res. 1988;14(7):479-89.

3. Cirillo R, Barone D, Franzone JS. Doxofylline, an antiasthmatic drug lacking affinity for adenosine receptors. Arch Int Pharmacodyn Ther. 1988;295:221-37.

4. Dolcetti A, Osella D, De Filippis G, Carnuccio C, Grossi E. Comparison of intravenously administered doxofylline and placebo for the treatment of severe acute airways obstruction. J Int Med Res. 1988;16(4):264-9.

5. Poggi R, Brandolese R, Bernasconi M, Manzin E, Rossi A. Doxofylline and respiratory mechanics. Short-term effects in mechanically ventilated patients with airflow obstruction and respiratory failure. Chest. 1989:96(4):772-8.

6. Cazzola M, Calzetta L, Barnes PJ, Criner GJ, Martinez FJ, Papi A, et al. Efficacy and safety profile of xanthines in COPD: a network meta-analysis. Eur Respir Rev. 2018;27(148)

7. Mennini F, Sciattella P, Marcellusi A, Marcobelli A, Russo A, Caputi A. Treatment plan comparison in acute and chronic respiratory tract diseases: an observational study of doxophylline vs. theophylline. Expert Rev Pharmacoecon Outcomes Res. 2017:17(5):503-10.

8. Calzetta L, Hanania NA, Dini FL, Goldstein MF, Fairweather WR, Howard WW et al. Impact of doxofylline compared to theophylline in asthma: a pooled analysis of functional and clinical outcomes from two multicentre, doubleblind, randomized studies (DOROTHEO 1 and DOROTHEO 2). Pulm Pharmacol Ther. 2018;53:20-6.

9. Margay SM, Farhat S, Kaur S, Teli HA. To study the efficacy and safety of doxophylline and theophylline in bronchial asthma. Journal of clinical and diagnostic research: JCDR. 2015:9(4):FC05-8.

10. Lal D, Manocha S, Ray A, Vijayan VK, Kumar R. Comparative study of the efficacy and safety of theophylline and doxofylline in patients with bronchial asthma and chronic obstructive pulmonary disease. J Basic Clin Physiol Pharmacol. 2015;26(5):443-51.

11. Rajanandh MG, Nageswari AD, llango K. Assessment of montelukast, doxofylline, and tiotropium with budesonide for the treatment of asthma: which is the best among the second-line treatment? A randomized trial. Clin Ther. 2015;37(2):418-26.

12. Rajanandh $M G$, Nageswari $A D$, llango K. Pulmonary function assessment in mild to moderate persistent asthma patients receiving montelukast, doxofylline, and tiotropium with budesonide: a randomized controlled study. Clin Ther. 2014;36(4):526-33.

13. Patel YA, Patel P, Bavadia H, Dave J, Tripathi CB. A randomized, open labeled, comparative study to assess the efficacy and safety of controller medications as add on to inhaled corticosteroid and longacting beta2 agonist in the treatment of moderate-to-severe persistent asthma. J Postgrad Med. 2010;56(4):270-4.

14. Bagnato GF. Tolerability of doxofylline in the maintenance therapy of pediatric patients with bronchial asthma. Eur Rev Med Pharmacol Sci. 1999;3(6):255-60.
15. Moher D, Shamseer L, Clarke M, Ghersi D, Liberati A, Petticrew M, et al. Preferred reporting items for systematic review and meta-analysis protocols (PRISMA-P) 2015 statement. Systematic reviews. 2015;4(1).

16. Schardt C, Adams MB, Owens T, Keitz S, Fontelo P. Utilization of the PICO framework to improve searching PubMed for clinical questions. BMC Med Inform Decis Mak. 2007;7:16.

17. Cazzola M, Calzetta L, Rogliani P, Page C, Matera MG. Impact of doxofylline in COPD: a pairwise meta-analysis. Pulm Pharmacol Ther. 2018:51:1-9.

18. Matera MG, Page C, Cazzola M. Doxofylline is not just another theophylline! Int J Chron Obstruct Pulmon Dis. 2017;12:3487-93.

19. Spina D, Page CP. Xanthines and phosphodiesterase inhibitors. Handb Exp Pharmacol. 2017;237:63-91.

20. Pavord ID, Beasley R, Agusti A, Anderson GP, Bel E, Brusselle G, et al. After asthma: redefining airways diseases. Lancet. 2018;391(10118):350-400.

21. Calzetta L, Rogliani P, Matera MG, Cazzola M. A systematic review with meta-analysis of dual bronchodilation with LAMA/LABA for the treatment of stable COPD. Chest. 2016;149(5):1181-96.

22. Cazzola M, Rogliani P, Calzetta L, Hanania NA, Matera MG. Impact of mucolytic agents on COPD exacerbations: a pair-wise and network metaanalysis. COPD. 2017;14(5):552-63.

23. Guyatt G, Oxman AD, Akl EA, Kunz R, Vist G, Brozek J, et al. GRADE guidelines: 1. Introduction-GRADE evidence profiles and summary of findings tables. J Clin Epidemiol. 2011;64(4):383-94.

24. Pedder H, Sarri G, Keeney E, Nunes V, Dias S. Data extraction for complex meta-analysis (DECiMAL) guide. Systematic reviews. 2016;5(1):212.

25. Li T, Puhan MA, Vedula SS, Singh S, Dickersin K. Ad hoc network meta-analysis methods meeting working $\mathrm{G}$. network meta-analysis-highly attractive but more methodological research is needed. BMC Med. 2011;9:79.

26. Calzetta L, Rogliani P, Ora J, Puxeddu E, Cazzola M, Gabriella Matera M. LABA LAMA combination in COPD: a meta-analysis on the duration of treatment. Eur Respir Rev 2016:0:1-11. https://doi.org/10.1183/16000617.0043-2016.

27. Cazzola M, Calzetta L, Rogliani P, Matera MG. Tiotropium formulations and safety: a network meta-analysis. Therapeutic Advances in Drug Safety 2016: 2042098616667304. https://doi.org/10.1177/2042098616667304.

28. Wallace BC, Dahabreh IJ, Trikalinos TA, Lau J, Trow P, Schmid CH. Closing the gap between methodologists and end-users: $R$ as a computational Back-end. J Stat Softw. 2012;49(5):1-15.

29. van Valkenhoef $G$, Lu G, de Brock B, Hillege H, Ades AE, Welton NJ. Automating network meta-analysis. Res Synth Methods. 2012;3(4):285-99.

30. Bangalore S, Messerli FH. Of statistical significance: "trends" toward significance and optimism bias. J Am Coll Cardiol. 2006;48(7):1471 author reply.

31. Jones PW, Beeh KM, Chapman KR, Decramer M, Mahler DA, Wedzicha JA. Minimal clinically important differences in pharmacological trials. Am J Respir Crit Care Med. 2014;189(3):250-5.

32. Guyatt GH, Oxman AD, Kunz R, Brozek J, Alonso-Coello P, Rind D, et al. GRADE guidelines 6. Rating the quality of evidence--imprecision. J Clin Epidemiol. 2011;64(12):1283-93.

33. Cazzola M, Rogliani P, Calzetta L, Matera MG. Triple therapy versus single and dual long-acting bronchodilator therapy in COPD: a systematic review and meta-analysis. Eur Respir J. 2018;52:6.

34. Kanazawa $\mathrm{H}$. Anticholinergic agents in asthma: chronic bronchodilator therapy, relief of acute severe asthma, reduction of chronic viral inflammation and prevention of airway remodeling. Curr Opin Pulm Med. 2006;12(1):60-7.

35. Matera MG, Calzetta L, Gritti G, Gallo L, Perfetto B, Donnarumma G, et al. Role of statins and mevalonate pathway on impaired HDAC2 activity induced by oxidative stress in human airway epithelial cells. Eur J Pharmacol. 2018;832:114-9.

36. GINA. Global Initiative for Asthma (GINA). Global strategy for asthma management and prevention. 2018. Available at: https:/ginasthma.org/ wp-content/uploads/2018/04/wms-GINA-2018-report-V1.3-002.pdf. Accessed 6 June 2019

\section{Publisher's Note}

Springer Nature remains neutral with regard to jurisdictional claims in published maps and institutional affiliations. 